\title{
Spatial and Temporal Independent Component Analysis Of Micro-Doppler Features
}

\author{
Victor C. Chen, Ph. D., US Naval Research Laboratory
}

Key Words: radar micro-Doppler, feature extraction, independent component analysis

\section{SUMMARY \& CONCLUSIONS}

Micro-Doppler features can be regarded as a unique signature of an object with movements and provide additional information for classification, recognition and identification of the object. Independent component analysis (ICA) can decompose micro-Doppler features into independent basis functions that represent salient physical movement attributes of the object. To study ICA of micro-Doppler features, we used a dataset generated by simulation of radar returned signals from rotating objects and tumbling objects. Fast ICA algorithm was used in our study to decompose micro-Doppler features into a set of spatial and temporal independent components. Spatial characteristics of the independent components combined with the corresponding temporal characteristics can be used to improve performance of classification, recognition and identification.

\section{INTRODUCTION}

Micro-motions, such as vibrations or rotations of an object or structures on the object, may induce additional frequency modulations on radar returned signal which generate sidebands about the object's Doppler frequency, called micro-Doppler [1]. Sources of micro-motions may be a rotor of a helicopter, a rotating antenna, natural mechanical oscillations in a bridge or a building, an engine-induced vibrating surface, or other causes. Electromagnetic backscattering from an object with micro-motion dynamics is subject to modulations in amplitude, phase, and polarization. The relationship between the object's micro-motions and the modulation characteristics constitutes distinguishable features in radar signatures.

Because micro-motions impose periodic and time-varying modulations onto the carrier frequency, to explore these features, time-frequency analysis [2] is used to provide information about these localized features in the joint time and frequency domain.

Micro-Doppler features can be regarded as a unique signature of an object that enables us to determine motion dynamic properties and provides additional information on the object that is complementary to existing methods. Thus, how to extract and analyze the salient micro-Doppler features becomes an important issue.

\section{ANALYSIS OF MICRO-DOPPLER FEATURES}

Principal component analysis (PCA) [3] is a best-known method for feature analysis that uses eigenvectors with the largest eigenvalues to obtain a set of basis functions such that the original function can be represented by a linear combination of these basis functions. The basis functions found by the PCA are uncorrelated, i.e., they cannot be linearly predicted from each other. However, higher order dependencies still exist in the PCA and, therefore, the basis functions are not properly separated.

In analyzing spatially localized micro-Doppler features, high-order relationship among the basis functions may contain important information. Thus, a feature analysis sensitive to high-order relationship is needed. Independent component analysis (ICA) is just such a method [4-6].

ICA was originally developed to separate mixed signals into independent components for blind source separation. Because it can be used to find independent physical attributes, another important application of the ICA is feature extraction. It decomposes a set of features into a basis whose components are statistically independent. ICA minimizes the statistical dependence between basis functions and searches for a linear transformation $W_{I C A}$ to express a set of features $X=\left(X_{1}\right.$, $\left.X_{2}, \ldots X_{\mathrm{M}}\right)$ as a linear combination of statistically independent basis functions $I C=\left(I C_{1}, I C_{2}, \ldots I C_{\mathrm{N}}\right)$, so that the transformed components

$$
I C=W_{I C A} X
$$

are independent, i.e., knowledge of the value of $I C_{\mathrm{i}}$ provides no information on the value of $I C_{\mathrm{j}}$ for $i \neq j$. Because there is no closed form solution for finding the matrix $W_{I C A}$, iterative algorithms have been used to search for the transformation matrix.

As we know, independent events must be uncorrelated, but uncorrelated events may not be independent. PCA only requires the components to be uncorrelated. However, ICA accounts for higher order statistics and, thus, is a more powerful feature representation than PCA. In fact, PCA similar to the Fourier analysis is basically a global component analysis, whereas ICA similar to the time-frequency or wavelet analysis is basically a localized component analysis.

\section{INDEPENDENT COMPONENT DECOMPOSITION OF 2-D FUNCTIONS}

ICA has been used to 2-D function analysis, such as natural image analysis and face recognition $[7,8]$. Some results show that ICA outperforms PCA, and others show that there is not much performance difference between ICA and 
PCA. As a matter of fact, if the global features such as width and length are more important, then they are more easily extracted by PCA than ICA. If features such as microDoppler features are more spatially localized, ICA is better than PCA.

(a)

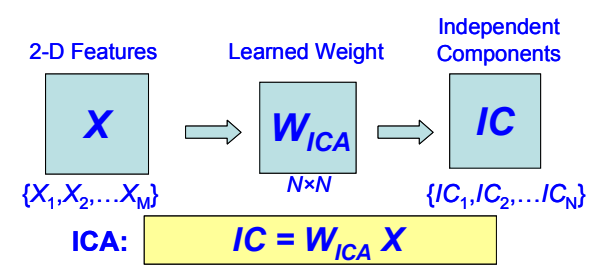

(b)

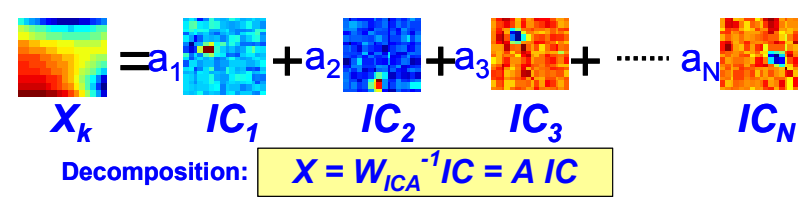

Figure 1 (a) An ICA block diagram, (b) IC decomposition.

ICA treats a 2-D function as a mixture of independent components. An architecture of ICA of a set of 2-D functions $X=\left(X_{1}, X_{2}, \ldots X_{\mathrm{M}}\right)$ is shown in Fig.1(a). ICA algorithm is an unsupervised learning that finds the weighting matrix $W_{I C A}$ such that a set of $N$ independent basis functions can be estimated by $I C=W_{I C A} X$. Thus, a 2-D function is formed by mixing the independent components together with a mixing matrix $A$ :

$$
X=A \cdot I C
$$

where $A=W_{I C A}{ }^{-1}$ and is a permuted version of $W_{I C A}{ }^{-1}$. Fig. 1(b) illustrates IC decomposition of a 2-D function into a set of independent basis components

$$
X_{k}=a_{1} I C_{1}+a_{2} I C_{2}+\ldots+a_{N} I C_{N}
$$

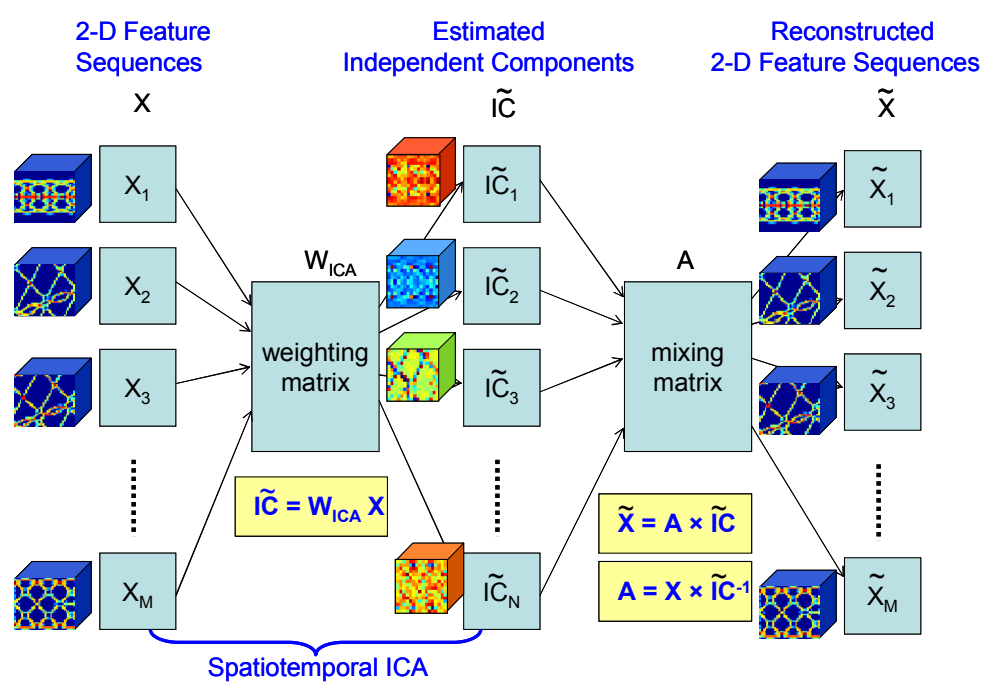

Figure 2 Spatiotemporal ICA.
The coefficients $\left(a_{1}, a_{2}, \ldots a_{\mathrm{N}}\right)$ of the linear combination of the independent components can be used in the independent component space to represent the 2-D function.

\section{SPATIAL AND TEMPORAL INDEPENDENT COMPONENT ANALYSIS OF 2-D FEATURES}

Most applications of the ICA are spatial analysis. Spatial ICA finds independent components of 2-D features over space, and represents a 2-D feature as a sum of the independent components $I C \mathrm{~s}$ :

$$
X_{k}(x, y)=\sum_{n=1}^{N} a_{n} \cdot I C_{n}(x, y)
$$

where $a_{n}$ is the weight of independent component $I C_{n}(x, y)$ and can be found from the given spatial feature $X_{k}(x, y)$ and the inverse of the independent components. Thus, the $I C_{n}(x, y)$ $(n=1,2, \ldots \mathrm{N})$ can be regarded as the basic building blocks of these 2-D features.

If a 2-D spatial feature varies over time, then temporal ICA may be used to find independent components over time. For many applications where 2-D features can be either spatially dependent, or temporally dependent, even both spatially and temporally dependent, ICA can be applied to spatial feature decomposition, temporal feature decomposition, and even the joint spatiotemporal feature decomposition $[6,7]$. The best features extracted from the spatial and temporal ICA may be used to improve the overall performance of the classification process.

To decompose a sequence of 2-D features over time, spatiotemporal ICA can maximize the degree of independence over space as well as time. Instead of using a set of 2-D spatial features, a set of 3-D space-time feature cubes, i.e., a stacked 2-D spatial features over time, are used to find a set of spatiotemporal independent components, as shown in Fig. 2.

$$
X_{k}(x, y, t)=\sum_{n=1}^{N} a_{n} \cdot I C_{n}(x, y, t)
$$




\section{SPACE-TIME MICRO-DOPPLER FEATURES}

Frequency modulation induced by micro-motion dynamics has both spatial and temporal characteristics. Fig. 3(a) shows an example of spatial micro-Doppler feature of a human gait process represented in the timefrequency domain [1]. Human gait is a complex motion behavior that comprises different movements of individual body parts.

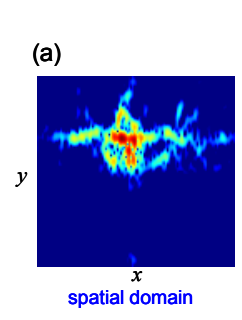

Spatial micro-Doppler feature of human gait
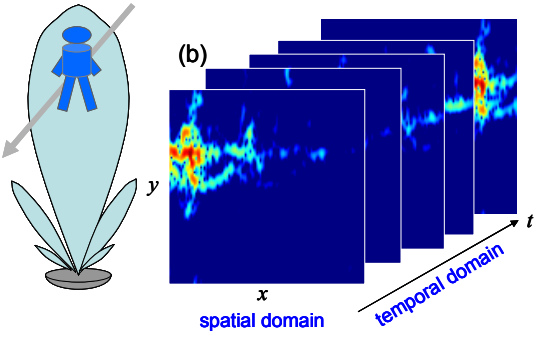

Temporal micro-Doppler feature of human gait
Figure 3. Human gait micro-Doppler features.

In the human gait micro-Doppler signature, the body Doppler shift and the Doppler modulation of the swinging arms can be clearly seen in Fig. 3 (a). The body Doppler shift is almost constant with a slightly saw-tooth shape because the body speeds up and slows down during the swing. However, the arm's micro-Doppler shift is a timevarying periodic curve. Doppler shift of one arm is higher and the other is lower than the body Doppler frequency shift. From the time information in the micro-Doppler feature, the swinging rate of the arm can be estimated. Fig. 3 (b) shows a temporal sequence of human gait microDoppler features. The movement of the body and the arms can be seen.

To illustrate applications of spatiotemporal ICA to micro-Doppler features, we simulate radar returns from two types of motion dynamics: rotation and tumbling. In our simulation, the point scatterer model is used for modeling the objects because it is simple compared to the EM prediction code simulation and is easy to observe the effect of micro-motions and separately study individual movements [9].

The geometry of the radar and a rotating object is illustrated in Figure 4(a). The radar coordinate system is defined by $(\mathrm{U}, \mathrm{V}, \mathrm{W})$, and the object local coordinate system is defined by $(x, y, z)$. Assume the azimuth and elevation angle of the object with respect to the radar coordinates $(\mathrm{U}, \mathrm{V}, \mathrm{W})$ are $\alpha$ and $\beta$, respectively.

Assume the object rotates about its axes $\mathrm{x}, \mathrm{y}$ and $\mathrm{z}$ with an angular velocity $\vec{\omega}=\left(\omega_{x}, \omega_{y}, \omega_{z}\right)^{T}$ or $\Omega=\|\vec{\omega}\|$ and the corresponding initial rotation matrix is defined by $\mathfrak{R}_{\text {Init }}$, thus, a point scatterer $\mathrm{P}$ initially located at $\vec{r}_{0}=\left(x_{0}, y_{0}, z_{0}\right)^{T}$ in the local coordinates $(\mathrm{x}, \mathrm{y}, \mathrm{z})$ is located at $\Re_{\text {Init }} \cdot \vec{r}_{0}$ and at time $t$ the scatterer $\mathrm{P}$ will move to $\vec{r}=\mathfrak{R}_{t} \mathfrak{R}_{\text {Init }} \cdot \vec{r}_{0}$, where $\mathfrak{R}_{t}$ is a rotation matrix. Thus, the rotation induced micro-Doppler modulation can be derived as

$$
f_{\text {Microboppler }}=\frac{2 f \Omega}{c}\left[\hat{\omega}^{\prime}\left(\hat{\omega}^{\prime} \sin \Omega t+I \cos \Omega t\right) \Re_{\text {Init }} \vec{r}_{0}\right]_{\text {radial }}
$$

where $\hat{\omega}^{\prime}$, called the skew symmetric matrix, is defined by the unit vector of the rotation $\vec{\omega}^{\prime}$, which is defined by $\vec{\omega}^{\prime}=\Re_{\text {Init }} \cdot \vec{\omega} /\|\vec{\omega}\| \quad[9]$.

From (6) we can see that the micro-Doppler induced by rotation is a sinusoidal function of $\Omega$ with an initial phase and amplitude depending on the initial position and the initial Euler angles $(\phi, \theta, \psi)$ of the scatterer.

In our simulation, assume the radar operates at $10 \mathrm{GHz}$ and an object, located at $(\mathrm{U}=1000 \mathrm{~m}, \mathrm{~V}=5000 \mathrm{~m}, \mathrm{~W}=$ $5000 \mathrm{~m}$ ), is rotating along the $\mathrm{x}, \mathrm{y}$ and $\mathrm{z}$ axes with a random initial Euler angles centered at $\left(\phi=45^{\circ}, \theta=45^{\circ}, \psi\right.$ $=45^{\circ}$ ) and angular velocity $\vec{\omega}=[\pi, \pi, \pi]^{T} \mathrm{rad} / \mathrm{sec}$. The object is designed as a cube that consists of eight point scatterers as illustrated in Fig. 4 (a). With a pulse repetition frequency (PRF) of 1000, a total of 2048 pulses was transmitted within $2.05 \mathrm{sec}$ of dwell time. An example of the spatial micro-Doppler induced by the rotation is shown in the Fig. 4 (b). From the micro-Doppler signature, the period of the rotation period can be calculated as $T=2 \pi /\|\vec{\omega}\|=1.16 \mathrm{sec}$.
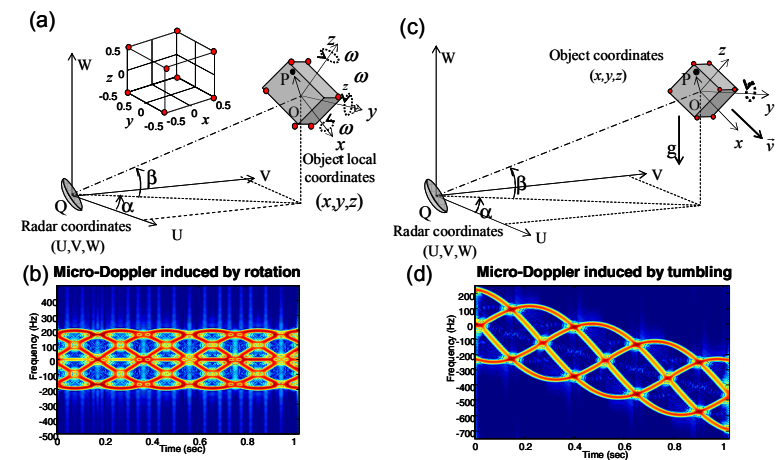

Figure 4 (a) A rotating object, (b) micro-Doppler induced by rotation, (c) a tumbling object, (d) micro-Doppler induced by tumbling.

The second object is a tumbling one. Tumbling consists of both translation and rotation motions as illustrated in Figure 4(c). Assume the object has an initial velocity $\mathrm{v}$ along the $\mathrm{x}$-axis and an acceleration of $\mathrm{g}=9.8$ $\mathrm{m} / \mathrm{s}^{2}$ due to gravity. At the same time, the object rotates along the y-axis. The azimuth and elevation angle of the origin $O$ of the object local coordinates with respect to the radar are $\alpha$ and $\beta$, respectively.

With the same radar parameters and the same object's model and location, assume the object is rotating along the $y$-axis with a random angular velocity centered at $\omega=2 \pi$ $\mathrm{rad} / \mathrm{sec}$ and with random initial Euler angles centered at $\phi=$ $45^{\circ}, \theta=45^{\circ}, \psi=45^{\circ}$. For simplicity, the initial velocity of 
the object is assumed to be $0 \mathrm{~m} / \mathrm{s}$. An example of the spatial micro-Doppler induced by the tumbling is shown in Figure 4(d).

\section{SPATIOTEMPORAL ICA OF MICRO-DOPPLER FEATURES}

The simulated micro-Doppler feature dataset described in Section 5 was used to study the spatiotemporal ICA. The dataset includes rotating objects with 50 different initial Euler angles and tumbling objects with 50 different initial Euler angles and angular velocities. In our study, the spatial ICA was first used to a set of spatial micro-Doppler features. Some selected samples of the spatial microDoppler features are shown in Fig. 5 (a) and 48 spatial ICs are obtained by the fixed-point fast ICA algorithm [10] as shown in Fig. 5 (b). Then, these 48 spatial ICs, served as the building blocks of the spatial micro-Doppler features induced by rotating objects and tumbling objects, can be used to reconstruct the micro-Doppler feature of a rotating or a tumbling object. Fig. 6 demonstrates the reconstructed micro-Doppler feature from the 48 spatial ICs.

To apply the spatiotemporal ICA, a set of space-time micro-Doppler feature sequences, each of them consists of 256 time samples of 16-by-16 spatial micro-Doppler features, was used. Weighting matrix was learned by the fast ICA algorithm using randomly selected feature sequences from the $100(16 \times 16 \times 256)$-micro-Doppler feature sequences in the dataset. Thus, a set of spatiotemporal independent components was found as the building blocks of the space-time feature functions as shown in Fig. 7, and any micro-Doppler feature sequence can be decomposed into the spatial and temporal feature basis functions. The wavelike temporal movement of the spatial features, as indicated in Fig. 7, added a new dimension to the micro-Doppler feature vector for improving the overall performance of classification, recognition and identification process. Fig. 8 shows the system diagram of the spatiotemporal ICA for microDoppler feature extraction. The spatial and temporal feature coefficients, which are the projections of a spacetime micro-Doppler feature into the spatiotemporal ICs, are the inputs to a classifier. Classifier estimates the optimal boundary in the feature space for classification, recognition and identification of an object. Here we will not discuss the issue of classifier in detail because it is beyond the scope of this paper.

\section{ACKOWLEDGEMENT}

This work was supported by ONR, through the NRL base program.

\section{(a) Samples of the Simulated Micro-Doppler Features}

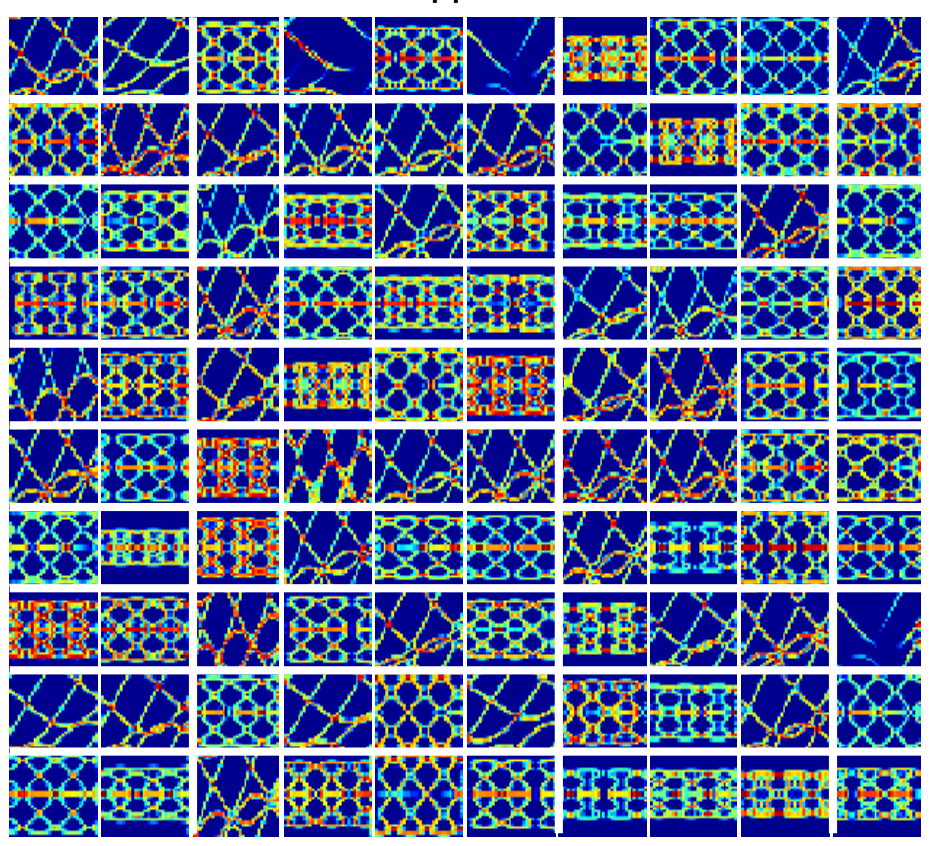

(b) Spatial ICs of Micro-Doppler Features

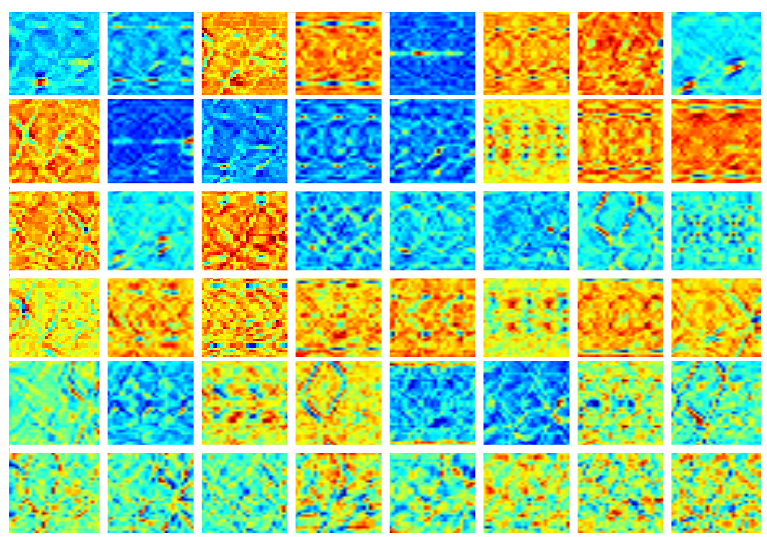

Figure 5. (a) Samples of spatial micro-Doppler features of rotating objects and tumbling objects, (b) spatial ICs estimated from the dataset. 

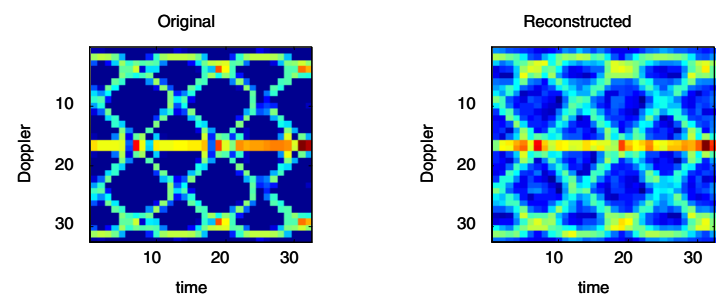

ICA Mixing Vector: A

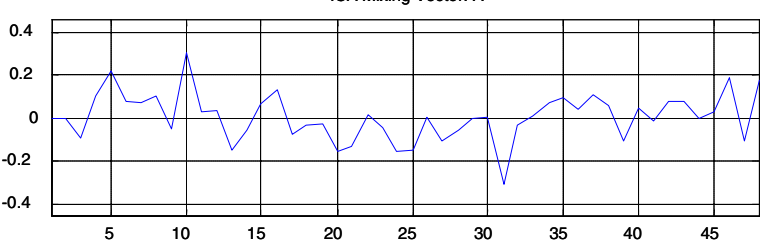

Figure 6 Reconstructed spatial micro-Doppler feature from 48 spatial ICs.

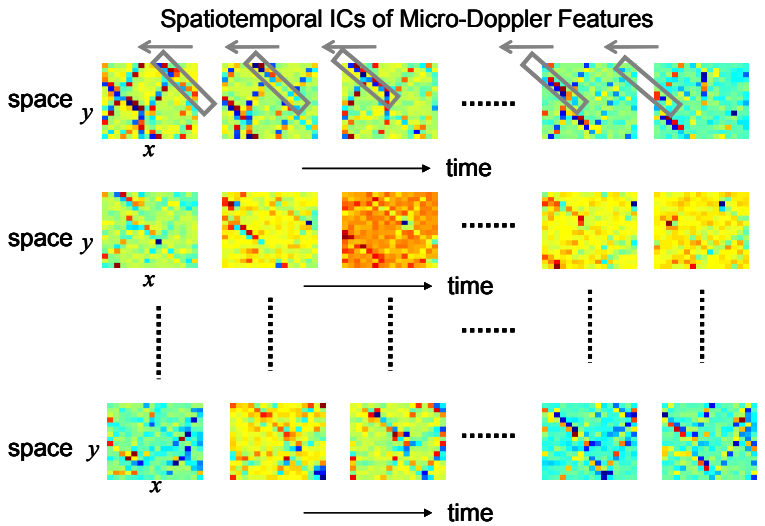

Figure 7 Spatiotemporal ICs estimated from the simulated dataset.

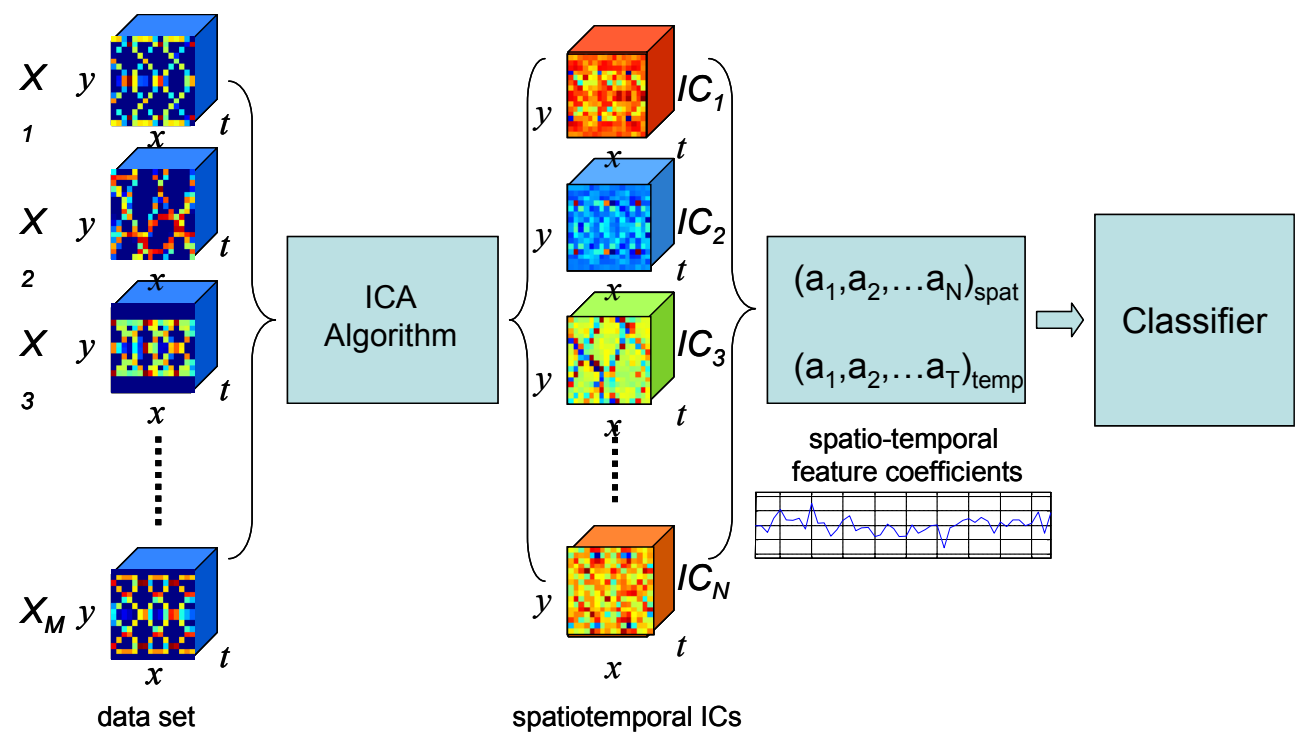

Figure 8 System diagram of the spatiotemporal ICA feature extraction and object classification.

\section{REFERENCES}

1. V.C. Chen and H. Ling, Time-Frequency Transforms for Radar Imaging and Signal Analysis, Artech House, 2002.

2. L. Cohen, Time-Frequency Analysis, Prentice Hall, 1995.
3. I.T. Jolliffe, Principal Component Analysis, New York: Springer, 1986.

4. A. Hyvarinen and E. Oja, "Independent component analysis: Algorithms and Applications", Neural Networks, 13(4-5), pp.411-430, 2000. 
5. A. Cichocki and S. Amari, Adaptive Blind Signal and Image Processing - learning algorithms and applications, John Wiley \& Sons, 2002.

6. J.V. Stone, Independent Component Analysis: A tutorial introduction, Bradford Book, 2004.

7. J.H. van Hateren and D.L. Ruderman, "Independent component analysis of natural image sequence yields spatio-temporal filters similar to simple cells in promary visual cortex", Proc. R. Soc. Lond. B. biol. Sci. Dec. 7, 265 (1412), pp.2315-2320, 1998.

8. M.S. Barlett, J.R. Movellan, and T.J. Sejnowski, "Face recognition by independent component analysis", IEEE Trans. on Neural Networs, vol.13, no.6, pp.14501464, 2002.

9. V.C. Chen, F. Li, S.-S. Ho, and H. Wechsler, "Analysis of micro-Doppler signatures", IEE Proc.Radar, Sonar Navig., vol.150, no.4, pp.271-276, 2003.

10. FastICA MATLAB package: http://www.cis.hut.fi/projects/ica/fastica/.
Victor C. Chen received Ph.D. degree in electrical engineering from Case Western Reserve University, Cleveland, Ohio in 1989. Since 1990 he has been with Radar Division, the U.S. Naval Research Laboratory in Washington DC and working on radar imaging, time-frequency applications to radar, and micro-Doppler analysis. He also serves as a program committee member and session chair for IEEE and SPIE conferences and served as an Associate Editor for IEEE Trans. on AES.

Dr. Chen has more than 100 publications in books, journals and proceedings including a book: Time-Frequency Transforms for Radar Imaging and Signal Analysis, published by Artech House in January 2002.

Address: Victor C. Chen, Code 5311, Radar Division, Naval Research Laboratory, 4555 Overlook Ave. S.W. Washington DC 20375, USA

vchen@radar.nrl.navy.mil 